\title{
Cross-Sectional Analysis of the Involvement of Interleukin- I7A in Diabetic Retinopathy in Elderly Individuals with Type 2 Diabetes Mellitus
}

\author{
Fuqiang Liu (iD) ${ }^{1-4}$ \\ Feng $\operatorname{Han}^{4,5}$ \\ Xiaoli Liu $^{4,5}$ \\ Lina Yang ${ }^{4,5}$ \\ Caixia Jiang ${ }^{4,5}$ \\ Chen Cui ${ }^{1-4}$ \\ Fang Yuan ${ }^{1-4}$ \\ Xin Zhang ${ }^{1-4}$ \\ Lei Gong ${ }^{\text {l-4 }}$ \\ Xinguo Hou ${ }^{1-4}$ \\ Yuan Liu ${ }^{1-4}$ \\ Li Chen ${ }^{1-4}$
}

'Department of Endocrinology, Qilu Hospital of Shandong University, Jinan, Shandong, 250012, People's Republic of China; ${ }^{2}$ Institute of Endocrine and Metabolic Diseases of Shandong University, Jinan, Shandong, 2500I2, People's Republic of China; ${ }^{3} \mathrm{Key}$ Laboratory of Endocrine and Metabolic Diseases, Shandong Province Medicine \& Health, Jinan, Shandong, 2500I2, People's Republic of China; ${ }^{4}$ Jinan Clinical Research Center for Endocrine and Metabolic Diseases, Jinan, Shandong, 250012, People's Republic of China; ${ }^{5}$ Department of Endocrinology, Zhangqiu District People's Hospital, Jinan, 250200,

People's Republic of China
Correspondence: Yuan Liu; Li Chen Email liuyuan198735@163.com; chenli3@medmail.com.cn
Background: To investigate the correlation between serum interleukin-17A (IL-17A) levels and diabetic retinopathy (DR) in elderly individuals with type 2 diabetes mellitus (T2DM). Methods: The study included 194 elderly patients (94 males and 100 females) with T2DM. Digital retinal photography as well as fundus fluorescein angiography was employed to distinguish between nonproliferative diabetic retinopathy (NPDR) and proliferative diabetic retinopathy (PDR). In addition, multiple logistic regression analysis was conducted to determine the correlation between serum IL-17A levels and DR status.

Results: The average age of the study cohort was $69.14 \pm 6.33$ years, of which $52.08 \%$ were male. The study participants with the highest IL-17A (Q4) levels had higher TC, DBP, and low-density lipoprotein cholesterol (LDL-C) values than those the other groups. Analysis using unadjusted and adjusted linear regression revealed that the effect size of 1.09 for DR in the unadjusted model indicates that IL-17A is associated with an increase of 1.09 in DR $(\mathrm{mmol} / \mathrm{L})(\beta 1.09,95 \%$ confidence interval (CI) 1.03, 1.16). Using the minimum-adjusted model (the model 2), as IL-17A increased, DR was higher by 1.11 ( $\beta 1.11,95 \%$ CI 1.04, 1.18 ). With the fully adjusted model (the model 3 ), for each additional IL-17A increase, DR was higher by 1.15 ( $\beta 1.15,95 \%$ CI $1.06,1.24)$.

Conclusion: Serum IL-17A levels are apparently positively correlated to DR in Chinese elderly individuals with T2DM.

Keywords: interleukin-17A, diabetic retinopathy, type 2 diabetes mellitus

\section{Background}

Type 2 diabetes mellitus (T2DM) is a global health problem that is strongly associated with high rates of morbidity and mortality. ${ }^{1}$ Diabetic retinopathy (DR) mainly results in prescient and imaginative blindness and visual impairment in working age individuals. The global prevalence of DR and severe DR has been estimated to be $35 \%$ and $10 \%$, respectively. ${ }^{2}$ DR has become a major public health problem in China. Because the current prevalence and risk factors for DR were mostly obtained from studies involving non-Asian populations, its application to Chinese populations remains uncertain. ${ }^{3}$ DR is also considered as a primary cause of decreased vision, visual loss and blindness in adult diabetic patients. ${ }^{4}$ In response to vascular damage, retinal vessels undergo angiogenesis, which lead to proliferative DR and blindness. ${ }^{5}$

Interleukin-17 (IL-17) is a cytokine family consisting of six structurally related isoforms, namely, IL-17A-F. IL-17A is considered the most important members of 
this family, and its upregulation has been associated with various autoimmune and chronic inflammatory diseases such as diabetes. ${ }^{6}$ IL-17A possesses strong proinflammatory activity, which is achieved by inducing the production of various pro-inflammatory cytokines, matrix metalloproteinases, and chemokines, and thus plays a central role in the etiology of inflammatory diseases. ${ }^{7}$ Some inflammatory factors include IL-17A, and IL-6 may come from the intestine. ${ }^{89}$ Recent studies have shown that anti-TCR combination therapy using anti-IL-6 and anti-IL -17 effectively increases the $\beta$ cell mass, whereas islet immune cell infiltrate only exhibited a slightly improvement in $\beta$ cell mass. Triple combination therapy effectively induced blood glucose homeostasis involving normalized serum C-peptide concentrations by controlling autoimmunity and improving anti-inflammatory effects. ${ }^{10}$ IL-17A may be involved in the pathological process of DR, but the relationship between them is still unclear. As the relationship between IL-17A and DR is complicated, more research is needed.

In our study, the main purpose was to investigate the relationship between IL-17A and DR. Therefore, by employing digital retinal photography and fundus fluorescein angiography in determining either nonproliferative DR (NPDR) or proliferative DR (PDR), we investigated the correlation between IL-17A and DR in Chinese elderly diagnosed with T2DM.

\section{Methods}

\section{Study Design}

This cross-sectional study was developed to establish the correlation between IL-17A and DR. This investigation used baseline IL-17A levels as the independent variable, whereas DR was the dependent variable.

\section{Study Population}

This cross-sectional study included 194 T2DM elderly individuals at the Qilu Hospital of Shandong University (Jinan, Shandong, China) from May 2019 to November 2019. We utilized the following patient exclusion criteria: (1) T1DM, lactic acidosis, diabetic ketoacidosis, or hyperosmolar nonketotic diabetic coma, or other special types of diabetes; (2) duration of diabetes of $<$ 1 year; (3) acute renal failure; (4) occurrence of diabetic foot or ulcers and other infectious diseases; (5) heart failure or acute cardio-cerebrovascular disease; and (6) severely impaired renal or liver function. Diabetes was diagnosed according to the 1999 World Health Organization criteria, ${ }^{11}$ ie, fasting blood glucose $(\mathrm{FBG}) \geq$ $7.0 \mathrm{mmol} / \mathrm{L}$ and/or 2-h postprandial blood glucose (PBG) $\geq 11.1 \mathrm{mmol} / \mathrm{L}$.

\section{Assessment of DR}

DR was assessed using color fundus photography and graded using the International Clinical DR Severity Scale. ${ }^{12}$ According to the standardized operation process, a digital retina camera is used to take high-quality fundus photos for both eyes after the pupils are dilated (CR-2 AF, CANON, Japanese). ${ }^{12}$ If necessary, fundus fluorescein angiography was conducted. Based on the Early Diabetic Retinopathy Study (ETDRS) Retinopathy Severity Score, ${ }^{14}$ digital retinal photography and fluorescein angiography were employed by professional ophthalmologists to assess and grade DR. ${ }^{13}$ The digital retinal camera captures 16.1 megapixel images and employed auto focus and shoot technology. Three undilated $45^{\circ}$ retinal photographs of each eye were captured by standard-trained technicians. ${ }^{14}$

\section{Clinical Data Collection}

We collected information about demographic characteristics and previous medical history through standard questionnaires. Anti-diabetes drugs consist of the following: insulin, insulin secretagogues, and other types ( $\alpha$-glucosidase inhibitors, thiazolidinedione, and metformin). Blood pressure (BP) was defined as three documented measurements (OMRON Model HEM-752 FUZZY, Omron Company, Dalian, China) $\geq 140 / 90 \mathrm{mmHg}$ at rest and using the left arm after sting for at least $5 \mathrm{~min}$, with the average reading employed in the analysis. History of hypertension and treatment with antihypertensive agents reported by the patient. After overnight fasting for at least $10 \mathrm{~h}$, venous blood samples were collected and used in measuring fasting blood glucose (FBG), fasting $\mathrm{C}$ peptide( $\mathrm{FCP})$, fasting insulin (FINS), alanine aminotransferase (ALT), aspartate aminotransferase (AST), blood urea nitrogen (BUN), creatinine (Cr), triglycerides (TGs), and high-density lipoprotein cholesterol (HDL-C) levels in an automatic analyzer (TOSHIBA TBA-40F, Toshiba, Japan). We used a modified method to directly measure high-density lipoprotein cholesterol (HDL-C). HbAlc was determined by high-performance liquid chromatography (BIO-RAD VARIANT II, Bio-Rad, USA). Venous blood samples were subjected to centrifugation at $3000 \mathrm{rpm}$ for $10 \mathrm{~min}$ to isolate sera, which were then aliquoted and stored at $-80^{\circ} \mathrm{C}$ until analysis of IL-17A levels. A multiplex 
magnetic bead-based antibody detection kit (Bio-Plex ${ }^{\circledR}$ suspension array system, Bio-Rad Laboratories Inc., Hercules, CA, USA), was employed for determination of IL-17A levels following the manufacturer's recommendations. This kit, for IL-17A, the inter-batch variation $(\% \mathrm{cv})$ is 2.4 , the inter-plate variation $(\% \mathrm{cv})$ is 1.4 , and the detection limit LLOQ is $2.44 \mathrm{pg} / \mathrm{mL}$. All patients' serum samples were measured in duplicate.

\section{Statistical Analysis}

The present study represented continuous variables using two approaches. We expressed the continuous variables of the normal distribution as the mean \pm standard deviation and displayed the continuous variables following a skewed distribution using the median (Q1Q4). Categorical variables were expressed as frequencies or percentages. In this study, we used one-way ANOVA test (normal distribution), $\chi^{2}$ test (categorical variables) or Kruskal-Wallis test (skew distribution) to test the differences between different IL-17A groups (quartiles). Data analysis was performed using two steps. Step 1: Single factor analysis and multiple linear regression analysis were used. This study generated three models: Model 1, which did not utilize covariate adjustment; Model 2, wherein adjusted was performed only for sociodemographic data; and Model 3, which was employed for variables in Model 2 and with covariate adjustment as shown in Table 1. Step 2: To resolve the nonlinear problem involving IL-17A and $\mathrm{DR}$, we conducted a generalized additive model with smooth curve fitting (penalized spline method). When nonlinearity was detected, we first computed for the inflection point with a recursive algorithm, and then generated a two-part linear regression for both sides of the inflection point. Using a log-likelihood ratio test, we then determined the best fit model using the $\mathrm{P}$ value. To ensure reliability of data analysis, we also performed sensitivity analysis. We converted IL-17A levels into categorical variables and computed for the $\mathrm{P}$ for the trend. This was performed to verify that the IL-17A results could be considered as a continuous variable and to assess non-linearity. We employed the $\mathrm{R}$ statistical package in all analyses (http://www. R-project.org, the $\mathrm{R}$ Foundation) and EmpowerStats (http://www.empowerstats.com, X\&Y Solutions, Inc., Boston, MA, USA). Differences with $P$ values $<0.05$ (two-sided) were deemed statistically significant.

\section{Results}

\section{Baseline Characteristics of Selected Subjects}

According to strict admission criteria, a total of 194 subjects were selected for final data analysis (for details, please refer to the selection and exclusion criteria). The baseline characteristics of the study participants are presented in Table 1 as IL-17A quartiles. The average age of the 194 selected participants was $69.14 \pm 6.33$ years, and approximately $52.08 \%$ of them were male. No statistically significant differences were observed based on diabetes mellitus (DM) duration, gender, total cholesterol (TC), HbA1c, low-density lipoprotein cholesterol (LDL-C), weight, or hypertension among various IL-17A groups (all $P$ values $>0.05$ ). The individuals in the group with the highest IL-17A (Q4) showed higher TC, LDL-C, DBP. Reverse patterns were observed using fasting $\mathrm{C}$ peptide (FCP).

\section{Univariate Analysis for DR}

The relevant findings generated by univariate analyses are presented in Table 2. Using univariate linear regression, we determined that gender, age, weight, FBG, HbA1c (\%), and LDL-C were not correlated with DR. We also observed that DM duration ( $\beta$ 1.09, 95\% CI 1.03, 1.14) and DBP ( $\beta$ 1.04, 95\% CI 1.01, 1.07) were correlated with DR. In contrast, univariate analysis revealed that (aspartate aminotransferase) AST/alanine aminotransferase (ALT) levels $(\beta 0.75,95 \%$ CI $0.29,1.94)$ had a positive correlation with DR.

\section{Results of Unadjusted and Adjusted Linear Regressions}

We then constructed three analysis models to assess the independent effect of IL-17A levels on DR (univariate and multivariate linear regression). Table 3 shows the effect sizes $(\beta)$ and $95 \%$ confidence intervals. Using the unadjusted model (model 1), the model-based effect size could explain the correlation between IL-17A and DR. For example, ie, the effect size of 1.09 for DR in the unadjusted model indicates that IL-17A is correlated to a 1.09 increase in DR $(\mathrm{mmol} / \mathrm{L})(\beta 1.09,95 \%$ CI 1.03, 1.16). In the minimum-adjusted model (model 2), as IL-17A increased, DR showed a 1.11 increase $(\beta 1.11,95 \% \mathrm{CI}$ $1.04,1.18$ ). Using the fully adjusted model (model 3 ) (adjusted for all covariates as shown in Table 1), for every additional increase in IL-17A, an increase in DR 


\begin{tabular}{|c|c|c|c|c|c|c|c|c|c|c|c|c|c|c|c|c|c|c|c|c|c|c|c|c|}
\hline 童 & & 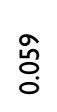 & 岕 & $\begin{array}{l}\text { 吉 } \\
\text { ô. }\end{array}$ & 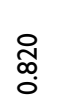 & $\frac{\vec{v}}{\hat{S}}$ & $\begin{array}{l}0 \\
\stackrel{o}{\infty} \\
0 \\
0\end{array}$ & م્ & $\begin{array}{c}\text { 岁 } \\
\text { o. } \\
0\end{array}$ & $\begin{array}{l}\hat{\hat{0}} \\
\text { Oे }\end{array}$ & ठ̃ & 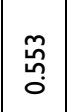 & 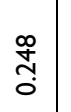 & $\begin{array}{l}\tilde{o} \\
o \\
o\end{array}$ & 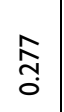 & $\frac{n}{\pi}$ & $\begin{array}{l}\overline{\bar{c}} \\
\dot{0}\end{array}$ & 旁 & $\begin{array}{c}\stackrel{ \pm}{\mathrm{m}} \\
0 \\
0\end{array}$ & 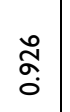 & 旁 & $\begin{array}{l}\vdots \\
\vdots \\
0\end{array}$ & 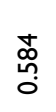 & $\begin{array}{c}\text { హ్ } \\
\text { ô }\end{array}$ \\
\hline ठ & $\dot{q}$ & 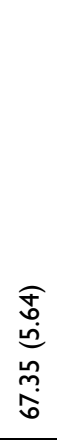 & $\begin{array}{l}\hat{\sigma} \\
\dot{0} \\
\sim \\
\hat{\sim} \\
\end{array}$ & $\begin{array}{l}\widehat{o} \\
\stackrel{0}{0} \\
\stackrel{0}{0} \\
\stackrel{0}{-}\end{array}$ & $\begin{array}{l}\frac{\sigma}{a} \\
\frac{\sigma}{a} \\
\frac{\sigma}{\infty} \\
0\end{array}$ & 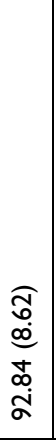 & $\begin{array}{l}\widehat{\sigma} \\
\dot{0} \\
\hat{m} \\
\dot{m} \\
\overline{0}\end{array}$ & $\begin{array}{l}\widehat{\widehat{n}} \\
\tilde{d} \\
\underline{d} \\
\infty \\
0 \\
\stackrel{0}{\underline{n}}\end{array}$ & $\begin{array}{l}\widehat{\varrho} \\
\stackrel{\dot{m}}{=} \\
\hat{\sigma} \\
\stackrel{\alpha}{a}\end{array}$ & 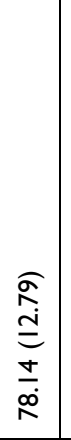 & 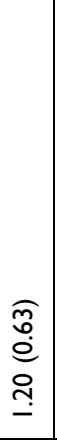 & 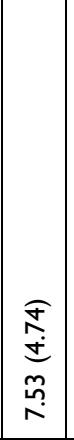 & 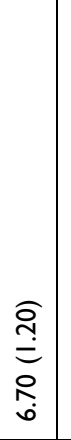 & 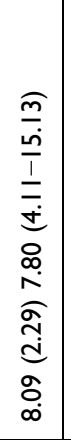 & 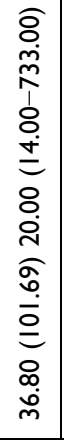 & 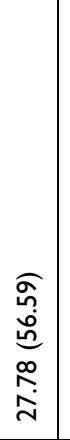 & $\begin{array}{l}\widehat{\bar{\sigma}} \\
\stackrel{c}{c} \\
\stackrel{\infty}{\underline{\square}}\end{array}$ & 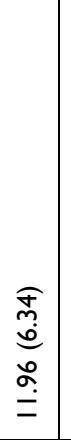 & 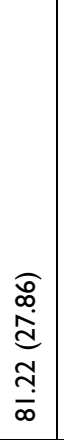 & 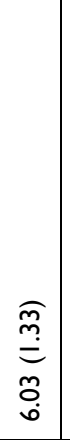 & 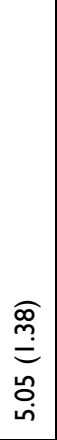 & 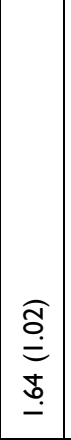 & 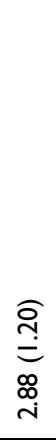 & $\begin{array}{l}\sigma \\
\tilde{m} \\
e \\
\stackrel{j}{j}\end{array}$ \\
\hline 0 & $\hat{f}$ & 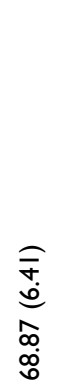 & $\begin{array}{l}\sigma \\
\frac{\sigma}{0} \\
0 \\
0 \\
\infty \\
\infty\end{array}$ & $\begin{array}{l}\widehat{\widehat{o}} \\
\stackrel{0}{0} \\
\stackrel{m}{-} \\
\underline{-}\end{array}$ & 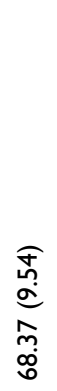 & $\begin{array}{l}\frac{\sigma}{\sigma} \\
\infty \\
m \\
\dot{\sigma} \\
\sigma\end{array}$ & 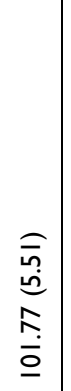 & 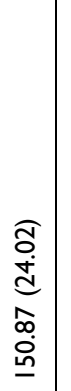 & 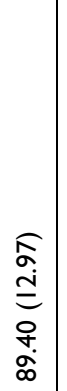 & 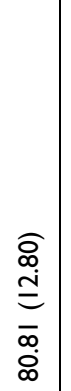 & 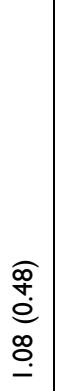 & $\begin{array}{l}\underset{\mathbb{N}}{\infty} \\
\stackrel{0}{0} \\
\frac{0}{\sigma}\end{array}$ & 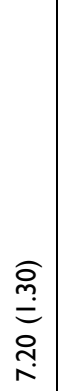 & 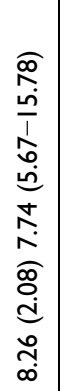 & 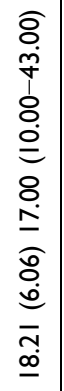 & $\begin{array}{l}\hat{\sigma} \\
\infty \\
\bar{\infty} \\
\underline{0}\end{array}$ & $\begin{array}{l}\underset{\mathbb{J}}{ \pm} \\
\stackrel{0}{0} \\
\underline{\Xi}\end{array}$ & 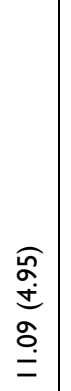 & 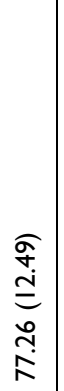 & $\begin{array}{l}\underset{\mathfrak{T}}{\leftrightarrows} \\
\frac{5}{0}\end{array}$ & 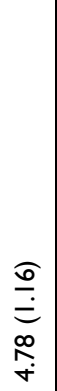 & $\begin{array}{l}\widehat{\infty} \\
o \\
0 \\
0 \\
0 \\
- \\
-\end{array}$ & 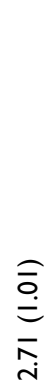 & $\begin{array}{l}\stackrel{f}{m} \\
\stackrel{m}{0} \\
\stackrel{m}{m}\end{array}$ \\
\hline
\end{tabular}

\begin{tabular}{|c|c|c|c|c|c|c|c|c|c|c|c|c|c|c|c|c|c|c|c|c|c|c|c|}
\hline & & 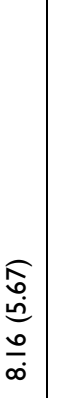 & $\begin{array}{l}\hat{\sigma} \\
\stackrel{0}{0} \\
\stackrel{0}{0}\end{array}$ & 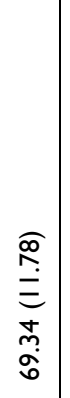 & 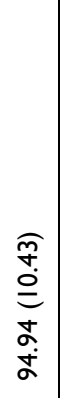 & $\left|\begin{array}{c}\tilde{\tilde{m}} \\
\infty \\
\tilde{\infty} \\
\tilde{n} \\
\tilde{c} \\
\underline{0}\end{array}\right|$ & 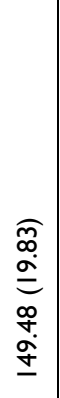 & 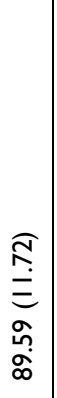 & $\begin{array}{l}\widehat{m} \\
\bar{\vdots} \\
\bar{\Xi} \\
0 \\
\dot{0} \\
\dot{\infty}\end{array}$ & $\begin{array}{l}\widehat{\tilde{n}} \\
\stackrel{0}{0} \\
\bar{c} \\
\underline{-}\end{array}$ & 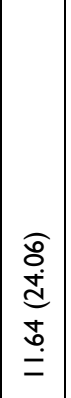 & 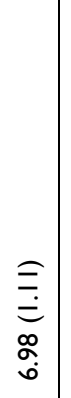 & 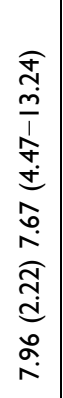 & 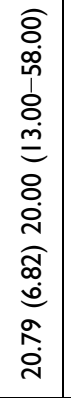 & $\begin{array}{l}\widehat{\cong} \\
\infty \\
\infty \\
\infty \\
\stackrel{\infty}{\simeq} \\
-\end{array}$ & 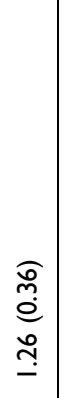 & 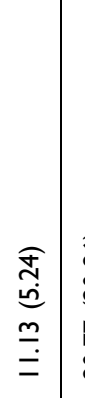 & $\begin{array}{l}\widehat{o} \\
\infty \\
\stackrel{d}{d} \\
\hat{N} \\
\stackrel{0}{0}\end{array}$ & $\begin{array}{l}\widehat{\widehat{\beta}} \\
\stackrel{0}{\oplus} \\
\stackrel{8}{8} \\
0 \\
0\end{array}$ & 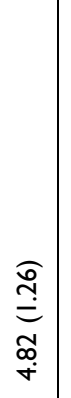 & 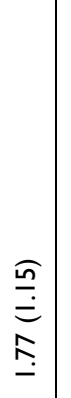 & $\begin{array}{l}\widehat{\sigma} \\
\dot{0} \\
\hat{e} \\
\dot{0} \\
\dot{i}\end{array}$ & 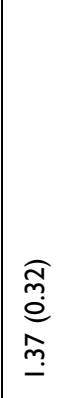 \\
\hline & & 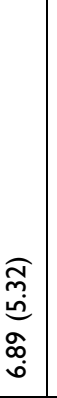 & 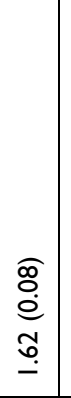 & $\begin{array}{c}\widehat{o} \\
\vdots \\
0 \\
\hat{a} \\
\hat{o} \\
\hat{0}\end{array}$ & 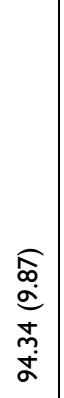 & 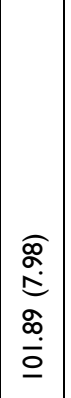 & $\begin{array}{l}\underline{\underline{0}} \\
\stackrel{d}{d} \\
\underline{\underline{0}} \\
\underline{\underline{n}}\end{array}$ & 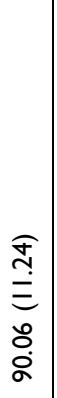 & 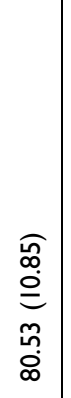 & $\begin{array}{l}\stackrel{o}{\rho} \\
\stackrel{0}{0} \\
o \\
\stackrel{\rho}{-}\end{array}$ & $\left|\begin{array}{c}\sigma \\
\sigma \\
0 \\
0 \\
\sigma \\
\sigma \\
\sigma\end{array}\right|$ & 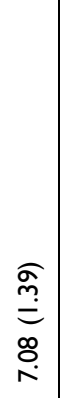 & 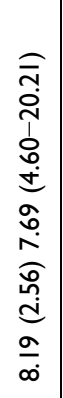 & 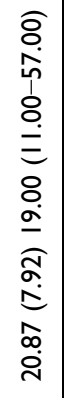 & 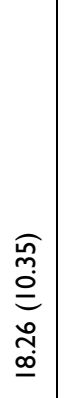 & 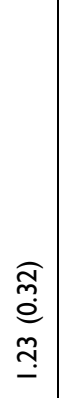 & 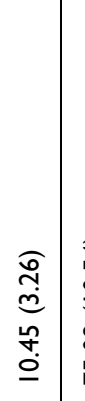 & 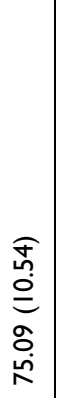 & 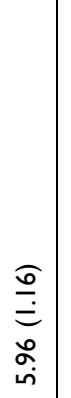 & 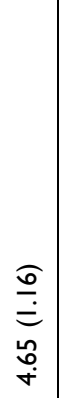 & 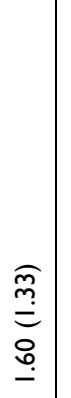 & 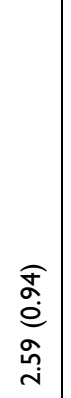 & 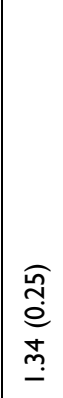 \\
\hline & & 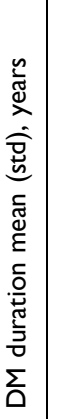 & 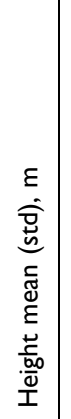 & 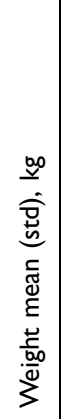 & 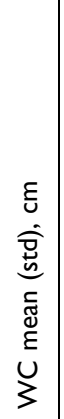 & 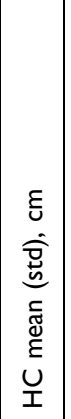 & 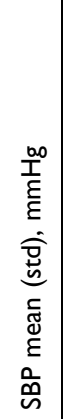 & 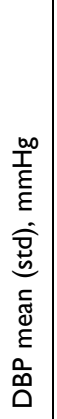 & 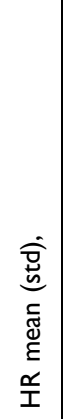 & 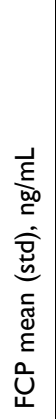 & 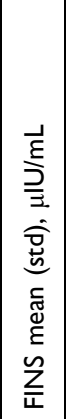 & 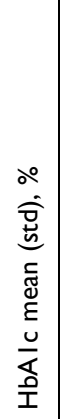 & 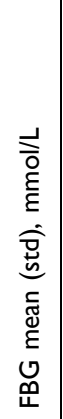 & 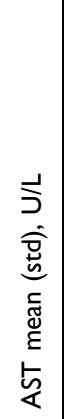 & 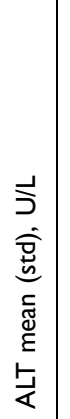 & 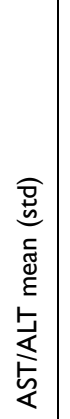 & 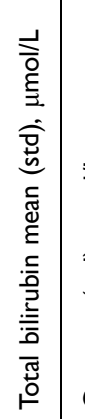 & 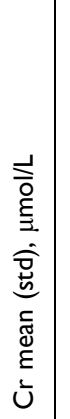 & 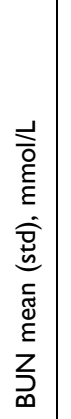 & 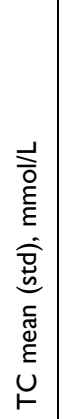 & 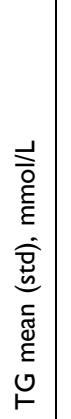 & 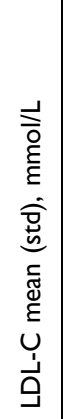 & 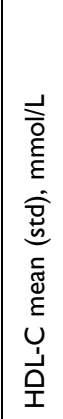 \\
\hline
\end{tabular}




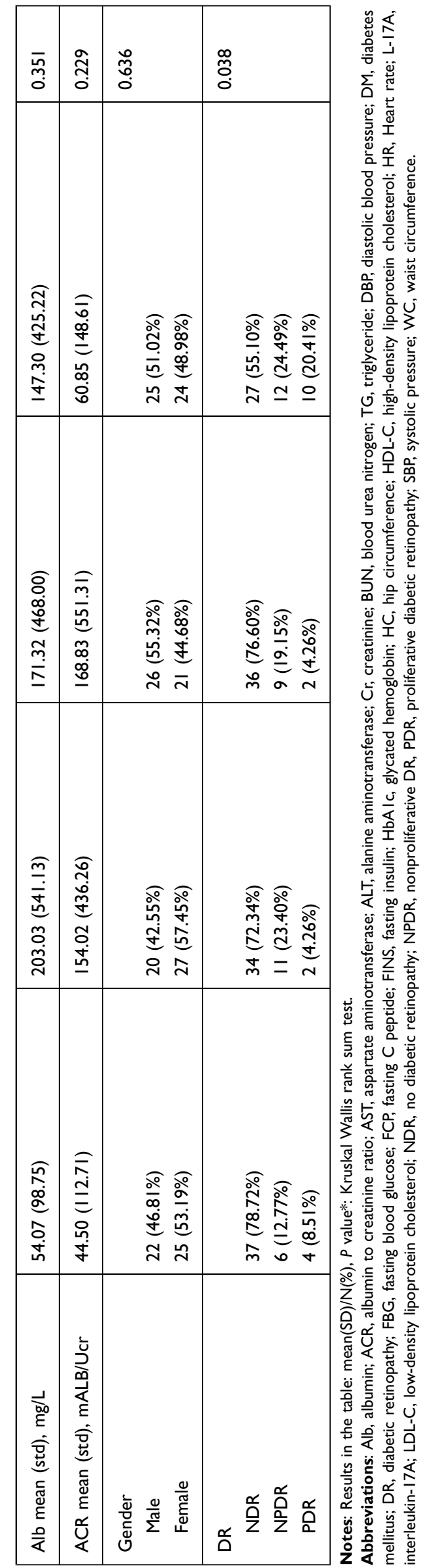

by $1.15(\beta 1.15,95 \%$ CI $1.06,1.24)$ was observed. For sensitivity analysis, we also converted IL-17A from a continuous variable into a categorical variable (quartile of IL-17A), and employed the $P$ for IL-17A profiling as a categorical variable with the fully adjusted model and the results were consistent with those when IL-17A was utilized as a continuous variable.

\section{The Results of the Association Between IL-I7A and DR}

In this study, we assessed the relationship between IL-17A and DR (Figure 1). The smooth curve and the findings of the generalized additive model revealed that the relationship between IL-17A and DR was generally linear after adjustment for age, sex, BMI, fasting C peptide (FCP), and SBP.

\section{Discussion}

Current DR treatment methods are mainly used in the advanced stages of the disease and may be related to serious side effects. Therefore, it is necessary to explore new diagnostic methods to identify the progression of DR earlier and monitor the effects of related treatments. Biochemical biomarkers refer to markers found in blood or other body fluids and tissues that can sensitively predict disease progression. ${ }^{15}$ These biomarkers may be a valuable tool for detecting the early stages of DR, identifying the people most likely to develop diabetic retinopathy and predicting treatment effect. ${ }^{16}$

This present cross-sectional study detected a significant difference in serum IL-17A levels between patients with and without DR. These findings indicate that DR is significantly correlated to increased odds of IL-17A after adjusting for gender, age, duration of diabetes, and other traditional diabetes factors. Some recent studies have shown that IL-17A is one of the key cytokines involved in the progression of diabetes complications. ${ }^{7,17,18}$ Their study confirmed that IL-17A plays an important role in fundus disease and blood vessel proliferation in diabetic mice, and found that $\mathrm{T}$ lymphocytes and neutrophils that express IL-17A in retinal blood vessels. ${ }^{19}$ The latest research suggests that intravitreal injection of IL$17 \mathrm{~A}$ will accelerate the progression of diabetic fundus lesions, while partial blockade of IL-17A receptors will delay the development of diabetes-mediated fundus lesions. ${ }^{20}$

Our previous research shows that the higher the serum IL-17A level, the higher the incidence and severity of DR. The underlying mechanism can be summarized as: (1) IL17A can trigger the destruction of the fundus vascular barrier as well as increase the degree of inflammation of 
Table 2 Univariate Analysis for Diabetic Retinopathy

\begin{tabular}{|c|c|c|c|}
\hline Covariate & Statistics & OR $(95 \% \mathrm{CI})$ & $P$-value \\
\hline Age (years) & $69.14 \pm 6.33$ & $1.00(0.95,1.05)$ & 0.9114 \\
\hline DM duration & $7.86 \pm 5.91$ & $1.09(1.03,1.14)$ & 0.0021 \\
\hline Height (m) & $1.62 \pm 0.08$ & $0.83(0.77, I .45)$ & 0.0759 \\
\hline Weight (kg) & $68.20 \pm 9.93$ & $1.00(0.97,1.03)$ & 0.8107 \\
\hline$W C(\mathrm{~cm})$ & $93.79 \pm 9.28$ & $1.00(0.97,1.03)$ & 0.9912 \\
\hline $\mathrm{HC}(\mathrm{cm})$ & $101.82 \pm 6.90$ & I.0I $(0.96,1.05)$ & 0.7120 \\
\hline \multicolumn{4}{|l|}{ Gender } \\
\hline Male & 94 (48.45\%) & 1.0 & \\
\hline Female & 100 (5I.55\%) & $1.15(0.62,2.15)$ & 0.6528 \\
\hline $\mathrm{SBP}(\mathrm{mmHg})$ & $150.48 \pm 21.85$ & $1.01(0.99,1.02)$ & 0.2369 \\
\hline $\mathrm{DBP}(\mathrm{mmHg})$ & $90.15 \pm 12.38$ & $1.04(1.01,1.07)$ & 0.0043 \\
\hline$H R$ & $79.94 \pm 11.84$ & $1.01(0.98,1.03)$ & 0.6646 \\
\hline $\mathrm{FCP}(\mathrm{ng} / \mathrm{mL})$ & $1.22 \pm 0.59$ & $1.43(1.02,2.85)$ & 0.0420 \\
\hline FINS & $9.49 \pm 13.93$ & $1.02(0.99,1.06)$ & 0.1369 \\
\hline HbAlc (\%) & $6.99 \pm 1.25$ & I.0I $(0.79$, I.29) & 0.9488 \\
\hline $\mathrm{FBG}(\mathrm{mmol} / \mathrm{L})$ & $8.13 \pm 2.26$ & $1.12(0.98,1.28)$ & 0.12240 .6786 \\
\hline AST (U/L) & $24.19 \pm 51.59$ & $1.00(0.99,1.01)$ & 0.9170 \\
\hline ALT (U/L) & $20.03 \pm 29.57$ & $1.00(0.99,1.01)$ & $0.554 I$ \\
\hline AST/ALT & $1.24 \pm 0.34$ & $0.75(0.29,1.94)$ & 0.9263 \\
\hline $\mathrm{Cr}(\mu \mathrm{mol} / \mathrm{L})$ & $78.39 \pm 19.28$ & $1.00(0.98,1.02)$ & 0.3247 \\
\hline BUN (mmol/L) & $6.02 \pm 1.35$ & $\mathrm{I} .12(0.89, \mathrm{I} .4 \mathrm{I})$ & 0.3505 \\
\hline $\mathrm{TC}(\mathrm{mmol} / \mathrm{L})$ & $4.83 \pm 1.23$ & I.I3 (0.88, I.45) & 0.4208 \\
\hline TG (mmol/L) & $1.67 \pm 1.13$ & $0.98(0.64,1.20)$ & 0.2218 \\
\hline LDL-C (mmol/L) & $2.71 \pm 1.03$ & I.2I $(0.89,1.64)$ & 0.5955 \\
\hline $\mathrm{HDL}-\mathrm{C}(\mathrm{mmol} / \mathrm{L})$ & $1.37 \pm 0.33$ & $1.29(0.51,3.26)$ & 0.8742 \\
\hline Alb (mg/L) & $|4| .96 \pm 416.47$ & $1.00(1.00,1.00)$ & 0.7957 \\
\hline $\mathrm{ACR}(\mathrm{mALB} / \mathrm{Ucr})$ & $105.03 \pm 360.7 \mid$ & $1.00(1.00,1.00)$ & \\
\hline
\end{tabular}

Abbreviations: Alb, albumin; ACR, albumin to creatinine ratio; AST, aspartate aminotransferase; ALT, alanine aminotransferase; BUN, blood urea nitrogen; TC, total cholesterol; $\mathrm{Cr}$, creatinine; DM, diabetes mellitus; HC, hip circumference; DBP, diastolic blood pressure; Cl, confidence interval; FBG, fasting blood glucose; FCP, fasting C peptide; FINS, fasting insulin; HbAIc, glycated hemoglobin; HDL-C, high-density lipoprotein cholesterol; HR, heart rate; OR, odds ratio; LDL-C, low-density lipoprotein cholesterol; SBP, systolic pressure; TG, triglyceride; WC, waist circumference.

Table 3 Correlation Analysis Between IL-I7A and DR Using Different Models

\begin{tabular}{|c|c|c|c|c|c|c|}
\hline \multirow[t]{2}{*}{ Variable } & \multicolumn{2}{|l|}{ Crude Model } & \multicolumn{2}{|l|}{ Model I ${ }^{\mathbf{a}}$} & \multicolumn{2}{|l|}{ Model II' } \\
\hline & $\beta(95 \% \mathrm{Cl})$ & $P$-value & $\beta(95 \% \mathrm{Cl})$ & $P$-value & $\beta(95 \% \mathrm{Cl})$ & $P$-value \\
\hline IL-I7A & $1.09(1.03,1.16)$ & 0.0056 & I.II (1.04, I.18) & 0.0026 & I.IS (1.06, I.24) & 0.0007 \\
\hline \multicolumn{7}{|l|}{ IL-I7A (quartile) } \\
\hline QI & Reference & & Reference & & Reference & \\
\hline Q2 & I.4I $(0.55,3.65)$ & 0.4727 & $1.26(0.47,3.34)$ & 0.6496 & $1.87(0.62,5.64)$ & 0.2671 \\
\hline Q3 & I.I3 $(0.43,2.99)$ & 0.8045 & $0.98(0.35,2.70)$ & 0.9623 & I.I9 (0.37, 3.82) & 0.7728 \\
\hline Q4 & $3.01(1.23,7.39)$ & 0.0159 & $3.23(1.25,8.32)$ & 0.0154 & $6.48(2.02,20.77)$ & 0.0017 \\
\hline$P$ for trend & 0.0159 & & 0.0154 & & 0.0017 & \\
\hline
\end{tabular}

Notes: a Model I adjusted for age, DM duration and gender; 'bmodel II adjusted for age, DM duration, sex, SBP, DBP, AST, ALT, GGT, AST/ALT, FBG, TC, LDL-C, HbAIc, ACR, FCP, Alb.

Abbreviations: $\mathrm{Cl}$, confidence interval; Alb, albumin, ACR, albumin to creatinine ratio, AST, aspartate aminotransferase; ALT, alanine aminotransferase; BUN, blood urea nitrogen, TC, total cholesterol; Cr, creatinine; DM, diabetes mellitus; DBP, diastolic blood pressure; FBG, fasting blood glucose; HbAIc, glycated hemoglobin; HDL-C, highdensity lipoprotein cholesterol; LDL-C, low-density lipoprotein cholesterol; SBP, systolic pressure. 


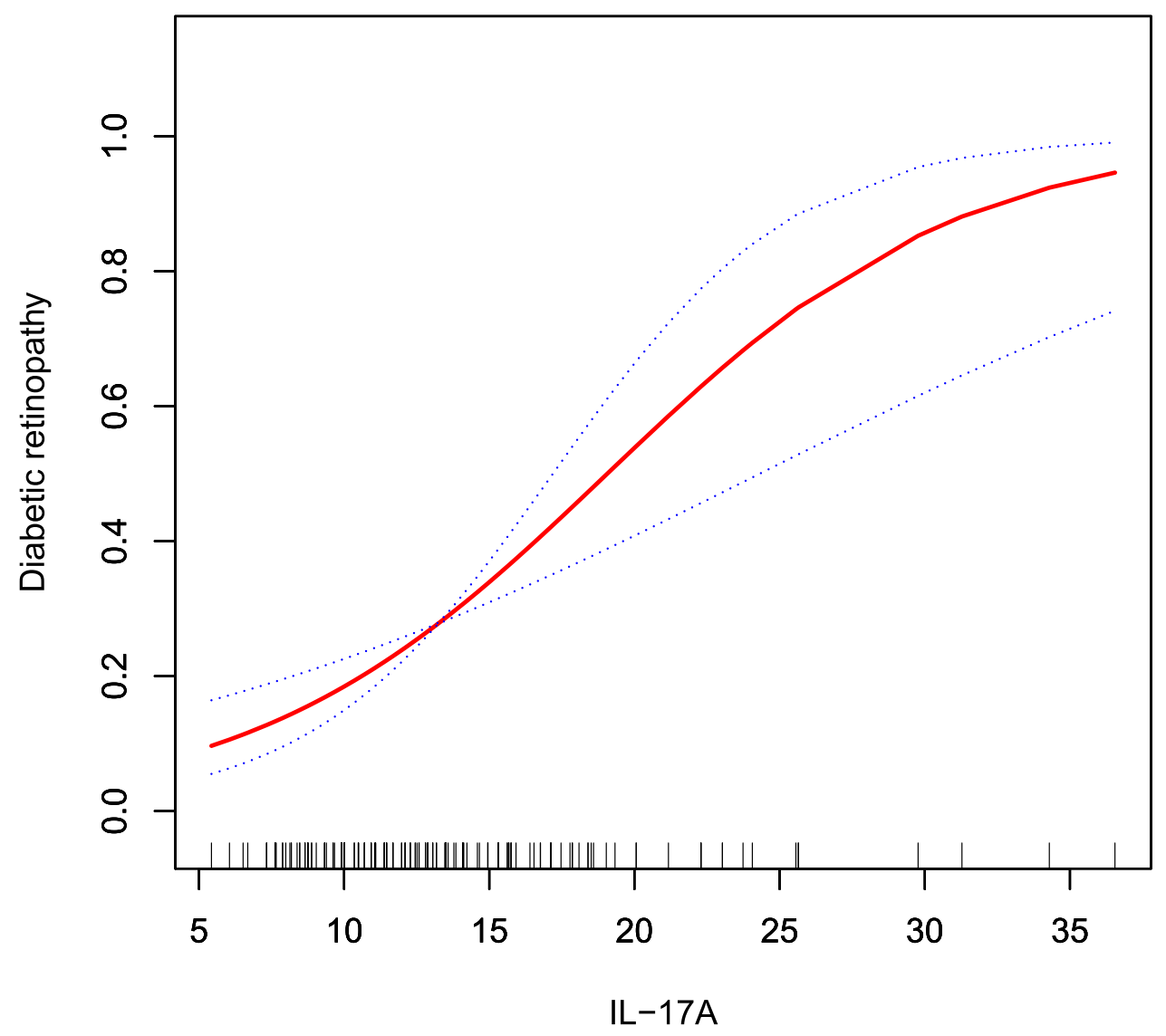

Figure I Association between interleukin-I7A (IL-I7A) and diabetic retinopathy (DR). A threshold, nonlinear association between IL-I7A and DR was determined $(P<0.00 \mathrm{I})$ in a generalized additive model (GAM). The solid red line shows the smooth curve fit between variables. Blue bands show the $95 \%$ of confidence interval from the fit. All were adjusted for age, gender, body mass index (BMI), SBP, and fasting $C$ peptide (FCP).

the fundus tissue by stimulating the secretion of inflammatory factors by vascular endothelial cells, ${ }^{21}$ and (2) IL$17 \mathrm{~A}$ triggers local retinal inflammation and immune response, which in turn accelerate DR progression. ${ }^{10}$

Global DR research studies have revealed that a longer T2DM duration, increasing age, higher HbA1c levels, and poorer blood pressure control were strongly correlated with DR. ${ }^{13,22}$ In this study, the longer the DM duration, the higher the prevalence and severity of DR, which is consistent with previous studies. ${ }^{23}$ Many previous studies have shown that FCP is related to $\mathrm{DR}^{24}$ An earlier study showed that after adjustment for confounding factors, C-peptide and insulin levels were significantly correlated with extent of DR in Latinos with type 2 diabetes. ${ }^{25}$ However, we did not observe a significant correlation between DR and FCP levels in the present study. Fasting plasma C peptide (OR, 1.43; 95\% CI 1.02 to $2.85 ; P=0.0420$ ) concentrations in these diabetic participants shows as a risk factor with retinopathy and its degree of severity. Our study population is mainly elderly diabetic patients, and renal function was generally reduced and plasma FCP levels were increased.
The main findings of this clinical study are as follows. (1) Thus far, this is a very meaningful study to investigate the independent association between serum IL-17A levels and DR in Chinese elderly T2DM patients. (2) This research helps to support the further research to looking for markers for diagnosis and treatment of diabetic retinopathy. (3) The correlation between IL-17A and DR may help predict the progressive pathology of fundus neovascularization in elderly patients with T2DM, and may participate in the development of T2DM.

Previous studies have shown that elevated levels of IL17A are correlated to the development of diabetic fundus lesions. ${ }^{6,7}$ In addition, serum IL-17A levels in individuals with PDR are significantly higher than those with NPDR or without retinopathy and controls. ${ }^{26-28}$ Actual clinical work shows that the current DR detection methods or grading standards cannot accurately reflect the degree of disease progression. ${ }^{19,26}$ This supports our hypothesis that IL-17A may be utilized as a sensitive predictor of retinopathy.

Our research also has some limitations: (1) In this study, we mainly included Chinese elderly patients with T2DM as the 
research objects. Therefore, there are certain deficiencies in the universality and extrapolation of the research. (2) The family history of T2DM patients is also an important baseline feature. However, in the data collected in this study, the patient's family history of T2DM was not collected. (3) Because individuals with T1DM were excluded from this study, as well as those with diabetic foot disease, acute renal failure, and severe infections, as well as those with kidney or liver disease or heart failure or acute cerebrovascular and cardiovascular disease, and those being treated with anti-gout drugs, the observations of this study may not be applicable to these people. (4) This investigation is a cross-sectional observational study and thus is susceptible to potential confounding factors. To minimize their effects, we employed rigorous statistical analysis.

\section{Conclusions}

This study provides evidence that suggests a novel link between IL-17A and DR in Chinese elderly individuals with T2DM. This correlation may also contribute to the pathogenesis of T2DM. Hence, serum IL-17A levels may be potentially employed as a biomarker for diabetic retinopathy, as well as serve as a novel therapeutic target for the treatment of diabetic fundus diseases.

\section{Abbreviations}

DM, diabetes mellitus; T2DM, Type 2 diabetes mellitus; ACR, albumin to creatinine ratio; Alb, albumin; HR, Heart Rate; BMI, Body Mass Index; WC, waist circumference; HC, hip circumference; BP, blood pressure; SBP, systolic pressure; DBP, diastolic blood pressure; BUN, blood urea nitrogen; $\mathrm{Cr}$, creatinine; ALT, alanine aminotransferase; AST, aspartate aminotransferase; GGT, glutamyl transpeptidase; TC, total cholesterol; TGs, triglycerides; HDL-C, high-density lipoprotein cholesterol; LDL-C, low-density lipoprotein cholesterol; FBG, fasting blood glucose; FCP, fasting C peptide; FINS, fasting insulin; HbAlc, glycated hemoglobin; IL-17A, interleukin-17A; DR, diabetic retinopathy; ETDRS, early diabetic retinopathy study; NDR, no diabetic retinopathy; NPDR, nonproliferative DR; PBG, Postprandial blood glucose; PDR, proliferative diabetic retinopathy; OR, odds ratio; GAM, generalized additive model; CI, confidence interval.

\section{Ethics Approval and Consent to Participate}

This study adhered to the principles of the Declaration of Helsinki and was approved by the Hospital Ethics Committee of the Qilu Hospital of Shandong University,
China, and written informed consent was obtained from the parents or guardians of all participants. All the authors have no objections to the ethical issues involved in the study and signed for confirmation.

\section{Acknowledgments}

This work was supported by the National Natural Science Foundation of China (Grant numbers 81400769, 81873632 and 81770818). This work also received support from the National Key R\&D Program of China (2018YFC1311801) and Cheeloo College of Medicine, Shandong University Graduate Education Innovation Plan (2020Y11).

\section{Author Contributions}

All authors made a significant contribution to the work reported, whether that is in the conception, study design, execution, acquisition of data, analysis and interpretation, or in all these areas; took part in drafting, revising or critically reviewing the article; gave final approval of the version to be published; have agreed on the journal to which the article has been submitted; and agree to be accountable for all aspects of the work.

\section{Funding}

There is no funding to report.

\section{Disclosure}

The authors declare that they have no competing interests.

\section{References}

1. Román-Pintos LM, Villegas-Rivera G, Rodríguez-Carrizalez AD, Miranda-Díaz AG, Cardona-Muñoz EG. Diabetic polyneuropathy in type 2 diabetes mellitus: inflammation, oxidative stress, and mitochondrial function. J Diabetes Res. 2016;2016:3425617. doi:10.1155/ 2016/3425617

2. Ting DSW, Cheung GCM, Wong TY. Diabetic retinopathy: global prevalence, major risk factors, screening practices and public health challenges: a review. Clin Experiment Ophthalmol. 2016;44:260-277. doi:10.1111/ceo.12696

3. He -B-B, Wei L, Gu Y-J, et al. Factors associated with diabetic retinopathy in Chinese patients with type 2 diabetes mellitus. Int J Endocrinol. 2012;2012:157940. doi:10.1155/2012/157940

4. Khan AA, Rahmani AH, Aldebasi YH. Diabetic retinopathy: recent updates on different biomarkers and some therapeutic agents. Curr Diabetes Rev. 2018;14:523-533. doi:10.2174/15733998136 66170915133253

5. Reddy S, Amutha A, Rajalakshmi R, et al. Association of increased levels of MCP-1 and cathepsin-D in young onset type 2 diabetes patients (T2DM-Y) with severity of diabetic retinopathy. $J$ Diabetes Complications. 2017;31(5):804-809. doi:10.1016/j.jdiaco mp.2017.02.017

6. Kumar P, Subramaniyam G. Molecular underpinnings of Th17 immune-regulation and their implications in autoimmune diabetes. Cytokine. 2015;71(2):366-376. doi:10.1016/j.cyto.2014.10.010 
7. Fores JP, Crisostomo LG, Orii NM, et al. Th17 pathway in recent-onset autoimmune diabetes. Cell Immunol. 2018;324:8-13. doi:10.1016/j.cellimm.2017.11.005

8. Zhu L, Sha L, Li K, et al. Dietary flaxseed oil rich in omega-3 suppresses severity of type 2 diabetes mellitus via anti-inflammation and modulating gut microbiota in rats. Lipids Health Dis. 2020;19(1):20. doi:10.1186/s12944-019-1167-4

9. Ye P, Xi Y, Huang Z, Xu P. Linking obesity with colorectal cancer: epidemiology and mechanistic insights. Cancers. 2020;12(6):1408. doi:10.3390/cancers 12061408

10. Jörns A, Ishikawa D, Teraoku $\mathrm{H}$, et al. Remission of autoimmune diabetes by anti-TCR combination therapies with anti-IL-17A or/and anti-IL-6 in the IDDM rat model of type 1 diabetes. BMC Med. 2020;18(1):33. doi:10.1186/s12916-020-1503-6

11. Alberti KGMM, Zimmet PZ. Definition, diagnosis and classification of diabetes mellitus and its complications. Part 1: diagnosis and classification of diabetes mellitus. Provisional report of a WHO consultation. Diabet Med. 1998;15:539-553. doi:10.1002/(sici)10969136(199807)15:7<539::aid-dia668>3.0.co;2-s

12. Ding J, Strachan MWJ, Reynolds RM, et al. Diabetic retinopathy and cognitive decline in older people with type 2 diabetes: the Edinburgh Type 2 Diabetes Study. Diabetes. 2010;59(11):2883-2889. doi: $10.2337 / \mathrm{db} 10-0752$

13. Zhang C, Wang S, Li M, Wu Y. Association between atherosclerosis and diabetic retinopathy in Chinese patients with type 2 diabetes mellitus. Diabetes Metab Syndr Obes. 2020;13:1911-1920. doi:10.2147/DMSO.S246497

14. Lee JC, Nguyen L, Hynan LS, Blomquist PH. Comparison of 1-field, 2-fields, and 3-fields fundus photography for detection and grading of diabetic retinopathy. J Diabetes Complications. 2019;33:107441. doi:10.1016/j.jdiacomp.2019.107441

15. Steinle JJ. Role of HMGB1 signaling in the inflammatory process in diabetic retinopathy. Cell Signal. 2020;73:109687. doi:10.1016/j. cellsig.2020.109687

16. Kaštelan S, Orešković I, Bišćan F, Kaštelan H, Gverović Antunica A. Inflammatory and angiogenic biomarkers in diabetic retinopathy. Biochem Med. 2020;30:030502. doi:10.11613/BM.2020.030502

17. Qiu AW, Liu QH, Wang JL. Blocking IL-17A alleviates diabetic retinopathy in rodents. Cell Physiol Biochem. 2017;41:960-972. doi:10.1159/000460514

18. Sindhu S, Akhter N, Arefanian H, et al. Increased circulatory levels of fractalkine (CX3CL1) are associated with inflammatory chemokines and cytokines in individuals with type-2 diabetes. J Diabetes Metab Disord. 2017;16(1):15. doi:10.1186/s40200-017-0297-3
19. Sigurdardottir S, Zapadka TE, Lindstrom SI, et al. Diabetes-mediated IL-17A enhances retinal inflammation, oxidative stress, and vascular permeability. Cell Immunol. 2019;341:103921. doi:10.1016/j. cellimm.2019.04.009

20. Qiu AW, Bian Z, Mao PA, Liu QH. IL-17A exacerbates diabetic retinopathy by impairing Müller cell function via Act1 signaling. Exp Mol Med. 2016;48:e280. doi:10.1038/emm.2016.117

21. Lindstrom SI, Sigurdardottir S, Zapadka TE, et al. Diabetes induces IL-17A-Act1-FADD-dependent retinal endothelial cell death and capillary degeneration. $J$ Diabetes Complications. 2019;33 (9):668-674. doi:10.1016/j.jdiacomp.2019.05.016

22. Parravano M, Oddone F, Boccassini B, et al. Functional retinal impairment in type 1 diabetic patients without any signs of retinopathy. Ophthalmic Res. 2013;50(2):108-112. doi:10.1159/ 000350412

23. Martinell M, Dorkhan M, Stålhammar J, et al. Prevalence and risk factors for diabetic retinopathy at diagnosis (DRAD) in patients recently diagnosed with type 2 diabetes (T2D) or latent autoimmune diabetes in the adult (LADA). J Diabetes Complications. 2016;30 (8):1456-1461. doi:10.1016/j.jdiacomp.2016.08.009

24. Chung JO, Cho DH, Chung DJ, Chung MY. Relationship between serum C-peptide level and diabetic retinopathy according to estimated glomerular filtration rate in patients with type 2 diabetes. $J$ Diabetes Complications. 2015;29:350-355. doi:10.1016/j.jdiacomp.2014.12.013

25. Kuo JZ, Guo X, Klein R, et al. Association of fasting insulin and $\mathrm{C}$ peptide with diabetic retinopathy in latinos with type 2 diabetes. BMJ Open Diabetes Res Care. 2014;2(1):e000027. doi:10.1136/ bmjdrc-2014-000027

26. Wang $\mathrm{C}$, Wang $\mathrm{L}$, Liu $\mathrm{J}$, et al. Irisin modulates the association of interleukin-17A with the presence of non-proliferative diabetic retinopathy in patients with type 2 diabetes. Endocrine. 2016;53 (2):459-464. doi:10.1007/s12020-016-0905-x

27. Chen H, Wen F, Zhang X, Su SB. Expression of T-helper-associated cytokines in patients with type 2 diabetes mellitus with retinopathy. Mol Vis. 2012;18:219-226.

28. Fatima N, Faisal SM, Zubair S, et al. Emerging role of Interleukins IL-23/ IL-17 axis and biochemical markers in the pathogenesis of type 2 diabetes: association with age and gender in human subjects. Int J Biol Macromol. 2017;105:1279-1288. doi:10.1016/j.ijbiomac.2017.07.155

\section{Publish your work in this journal}

Diabetes, Metabolic Syndrome and Obesity: Targets and Therapy is an international, peer-reviewed open-access journal committed to the rapid publication of the latest laboratory and clinical findings in the fields of diabetes, metabolic syndrome and obesity research. Original research, review, case reports, hypothesis formation, expert opinion and commentaries are all considered for publication. The manuscript management system is completely online and includes a very quick and fair peer-review system, which is all easy to use. Visit http://www.dovepress.com/testimonials.php to read real quotes from published authors. 